\title{
Impact of Thoracic Endovascular Repair on Pulsatile Aortic Strain in Acute Type B AorticD issection
}

\section{Preliminary Results}

Foeke J.H. Nauta, MD, PhD ${ }^{1,2^{*}}$, Guido H.W. van Bogerijen, MD, PhD ${ }^{1,2}$, Michele Conti, PhD ${ }^{3}$, Chiara Trentin, $\mathrm{PhD}^{4}$, Frans L. Moll, MD, PhD ${ }^{2}$, Joost A. Van Herwaarden, MD, PhD², Ferdinando Auricchio, $\mathrm{PhD}^{3,4}$, Santi Trimarchi, MD, PhD ${ }^{1}$

\author{
${ }^{1}$ Thoracic Aortic Research Center, Vascular Surgery, IRCCS Policlinico San Donato, San Donato Milanese, Italy \\ ${ }^{2}$ Department of Vascular Surgery, University Medical Center Utrecht, Utrecht, Netherlands \\ ${ }^{3}$ Structural Mechanics Division, Department of Civil Engineering and Architecture, University of Pavia, Italy \\ ${ }^{4}$ CESNA Center for Advanced Numerical Simulations, Istituto Universitario di Studi Superiori di Pavia (IUSS), Pavia, Italy
}

\begin{abstract}
Background: The impact of thoracic endovascular aortic repair (TEVAR) on pulsatile aortic strain remains undetermined in patients with Type $B$ aortic dissection (TBAD). Therefore, we quantified pulsatile aortic strain in TBAD patients and control subjects.

Methods: We retrospectively analyzed two TBAD patients from our database with cardiac-gated computed tomography angiography imaging available before and after TEVAR and two control subjects (67- and 76-year-old males). Patient 1 (54-year-old female) presented with acute TBAD, and Patient 2 (55-year-old male) had Marfan syndrome and ruptured acute TBAD. Custom-developed software was used to compute aortic length, diameter, and area during the cardiac cycle. Pulsatile strain was calculated as systolic increments of length and circumference divided by corresponding diastolic values.

Results: Before TEVAR, pulsatile longitudinal strain of the thoracic aorta was lower in TBAD patients (1.4-1.7\%) than in control subjects (2.1-4.5\%). After TEVAR, pulsatile longitudinal strain increased proximal to the stentgraft by $65 \%$ in the arch of Patient 1 and by $70 \%$ in the ascending aorta of Patient 2. Pulsatile circumferential
\end{abstract}

Published by Science International Corp. ISSN 2325-4637

Fax +1 2037853552

E-Mail: aorta@scienceinternational.org

http://aorta.scienceinternational.org strain was elevated in false lumen patency (4.4-6.2\%) compared with thrombosed false lumen $(1.4-2.1 \%)$ or control subjects (0.9-3.3\%). Following TEVAR, circumferential measurements within stented segments were deemed unreliable due to artifacts.

Conclusions: TEVAR led to a considerable increase of pulsatile longitudinal strain proximal to the stent-grafts, and TBAD was associated with longitudinally stiffer aortas, which may be part of the pathophysiology of TEVAR-related complications such as retrograde dissection and aneurysmal dilatation. These preliminary data call for larger prospective studies.

Copyright @ 2017 Science International Corp.

Key Words

Type B aortic dissection - Thoracic endovascular aortic repair • Aortic strain

\section{Introduction}

Thoracic endovascular aortic repair (TEVAR) has been successfully adopted to treat complicated Type B aortic dissection (TBAD) [1]. However, major
* Corresponding Author:

Foeke J.H. Nauta, MD, PhD Vascular Surgery

Thoracic Aortic Research Center

IRCCS Policlinico San Donato, Piazza Malan 2 20097, San Donato Milanese, Milan, Italy Tel: +39 02 52774344; Fax: +39 02 52774383; E-Mail: foekenauta@gmail.com 
TEVAR-related complications are widely reported with retrograde or antegrade dissection, typically originating at the proximal or distal end of the device, with rupture being the most lethal [2-4]. Unfortunately, the mechanisms of these TEVAR-related complications are mostly unknown. Changes in aortic dynamics during the cardiac cycle, induced by a mismatch between the stent-graft and the aorta, are suggested to play a key role $[2,5]$. However, little is known about deformations of the dissected aortic wall, and few studies have reported strain after endovascular repair [6].

Biomechanical studies show that current thoracic stent-grafts are about 125 times stiffer than the adult thoracic aorta (55.2 MPa vs. 0.44 MPa) [7, 8]. Implantation of such stiff devices may underlie reported cases of TEVAR-induced acute hypertension and cardiac remodeling $[9,10]$. However, the exact nature of TEVAR-induced local changes in aortic wall strain remains to be determined. While most dynamic imaging studies have focused on aortic area or diameter changes during the cardiac cycle [11-13], the importance of longitudinal strain should be not underestimated, as it is predominantly the longitudinal axis that fails in the event of aortic dissection [14-16]. During each cardiac contraction, the heart pulls the aortic root downward, resulting in longitudinal strain of about 7-9\% in the ascending aorta $[15,17]$. Implantation of a rigid stent-graft in the proximal descending aorta might stiffen that segment, potentially forcing the ascending aorta and aortic arch to overstretch during each heartbeat, as we observed in a study of eight aneurysm patients managed with TEVAR (unpublished data). Such elevated wall stress has been associated with increased risk of aortic dissection [15] and aneurysm growth [5].
These considerations led us to quantify pulsatile aortic strain in both longitudinal and circumferential directions in acute TBAD patients before and after TEVAR as well as control subjects. For this purpose, we used a semi-automatic technique to post-process cardiac-gated computed tomography angiography (CTA) image data.

\section{Materials and Methods}

For this retrospective study, we included patients with acute TBAD enrolled in our imaging database who underwent cardiac-gated CTA before and after TEVAR at the University of Utrecht, The Netherlands, which resulted in a limited number of patients $(n=2)$. Two patients without thoracic aortic disease served as control subjects (Table 1). The local ethical review board evaluated the study protocol; formal approval was given, and informed consent was waived.

\section{Patient Population}

Patient 1 was a 54-year-old female with acute TBAD and thrombosed false lumen (Figure $1 \mathrm{~A}$ ). This patient underwent TEVAR using two Bolton Relay (Bolton Medical Inc, Sunrise, FL) stent-grafts (Figure 1B). The proximal landing zone was just distal to the left common carotid artery, covering the left subclavian artery (LSA), with distal extension $10 \mathrm{~cm}$ above the celiac bifurcation. Stent-graft oversizing was $8 \%$. A postoperative cardiac-gated CTA was conducted 3 weeks later, which was used for this study. During 5 years of follow-up, no endoleaks or other stent-graft-related

Table 1. Patient and procedural characteristics.

\begin{tabular}{|c|c|c|c|c|c|c|}
\hline $\mathrm{Pt}$ & $\begin{array}{l}\text { Age (Years), } \\
\text { Gender }\end{array}$ & Aortic Disease & Dissection Extension & Stent-graft Types & $\begin{array}{l}\text { Stent-graft } \\
\text { Sizes }(\mathrm{mm})\end{array}$ & $\begin{array}{l}\text { Stent-graft Length } \\
\text { in situ ( } \mathrm{mm})\end{array}$ \\
\hline 1 & $54, F$ & Acute TBAD & $\begin{array}{l}5 \mathrm{~cm} \text { distal from the LSA } \\
\text { to the aortic bifurcation }\end{array}$ & Bolton Relay & $\begin{array}{l}36-36-200 \\
36-36-200\end{array}$ & 174 \\
\hline 2 & $55, M$ & $\begin{array}{l}\text { Ruptured acute TBAD, } \\
\text { Marfan syndrome }\end{array}$ & $\begin{array}{l}3 \mathrm{~cm} \text { distal from the LSA } \\
\text { into right iliac artery }\end{array}$ & Medtronic Captivia & $\begin{array}{l}34-34-200 \\
36-36-150\end{array}$ & 235 \\
\hline 3 & $66, M$ & None & - & - & - & - \\
\hline 4 & $75, M$ & None & - & - & - & - \\
\hline
\end{tabular}

$\mathrm{LSA}=$ left subclavian artery; $\mathrm{TBAD}=$ Type $\mathrm{B}$ aortic dissection 


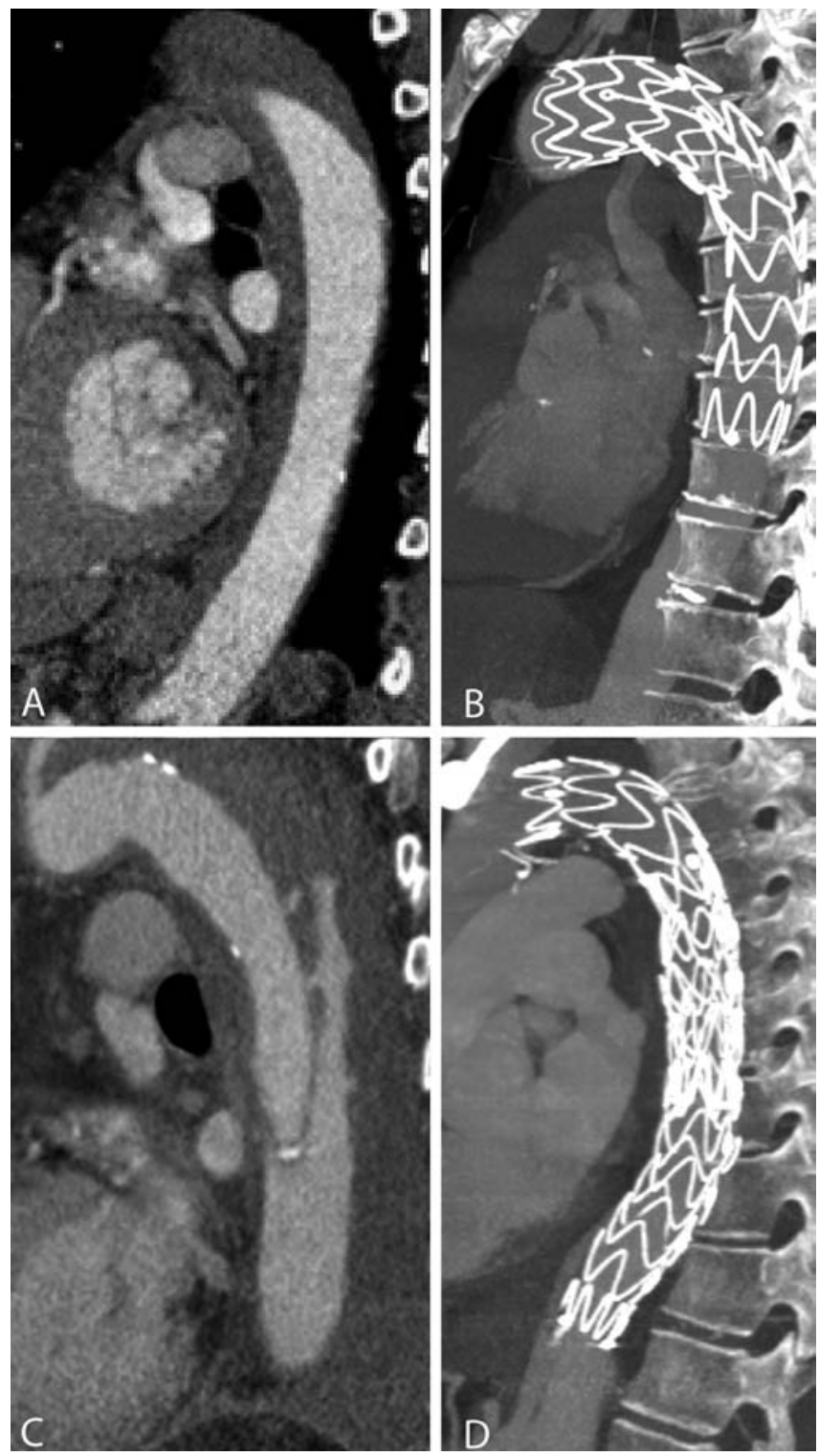

Figure 1. Panels $A-D$. Computed tomography angiography (CTA) imaging showing the acute type $B$ aortic dissection (TBAD) of Patient 1 (Panel $A$ ) before and (Panel $B$ ) after thoracic endovascular aortic repair (TEVAR) and the ruptured acute TBAD of Patient 2 (Panel $C$ ) before and (Panel $D$ ) after TEVAR.

\section{complications were noted.}

Patient 2 was a 55-year-old male with Marfan syndrome with acute TBAD and patent false lumen from the mid-descending aorta to the iliac arteries. This patient was initially managed with medical therapy. Six days after the first acute event, he developed a contained rupture of the proximal descending aorta and was managed with emergency TEVAR (Figure 1C).
Two Medtronic Captivia (Medtronic Vascular, Santa Rosa, CA) stent-grafts were implanted, with extension distal from the LSA to just proximal to the celiac bifurcation (Figure 1D). The stent-graft diameter was oversized by $40 \%$ considering the ruptured aorta and hypotension [18] found during examination as well as the unavailability of smaller devices in the acute setting in our hospital. The cardiac-gated CTA conducted at the second presentation and 3 days postoperatively were used for this study. After 1 month, CTA imaging revealed a pseudoaneurysm caused by aortic perforation of a proximal bare stent strut, as previously reported [19]. A proximal stent-graft extension was implanted (32-32-164 mm), landing just distal to the left common carotid artery and covering the origin of the LSA. No endoleaks or other stent-graft-related complications occurred during 5 years of follow-up, with complete thrombosis of the false lumen and subsequent positive remodeling of the aorta.

Patients 3 and 4 were both males (67- and 76-yearsold) with no visible evidence of thoracic aortic disease and no medical history of aortic surgery or connective tissue disorder.

\section{Workflow from Data Collection to Strain Quantification}

The workflow for this study is illustrated in Figure 2 and described stepwise below.

1. Image acquisition and data collection: Basic input data was a dicom set of cardiac-gated CTA images obtained during eight phases of the cardiac cycle. These dynamic images were obtained with a 256-row multislice CT system (Philips Medical System, Best, The Netherlands) and imported into 3Mensio software (3Mensio Medical Imaging, Bilthoven, The Netherlands) for analysis. A three-dimensional (3-D) scan volume was acquired during all eight phases, for which maximum (end-systolic) and minimum (end-diastolic) measurements were acquired. Patients were included in the study only if image acquisition was accomplished successfully and image quality was considered adequate. Pixel spacing and slice thickness were similar for all patients and are described in Supplemental Table 1 (see supplemental Table 1 at http://dx.doi. 

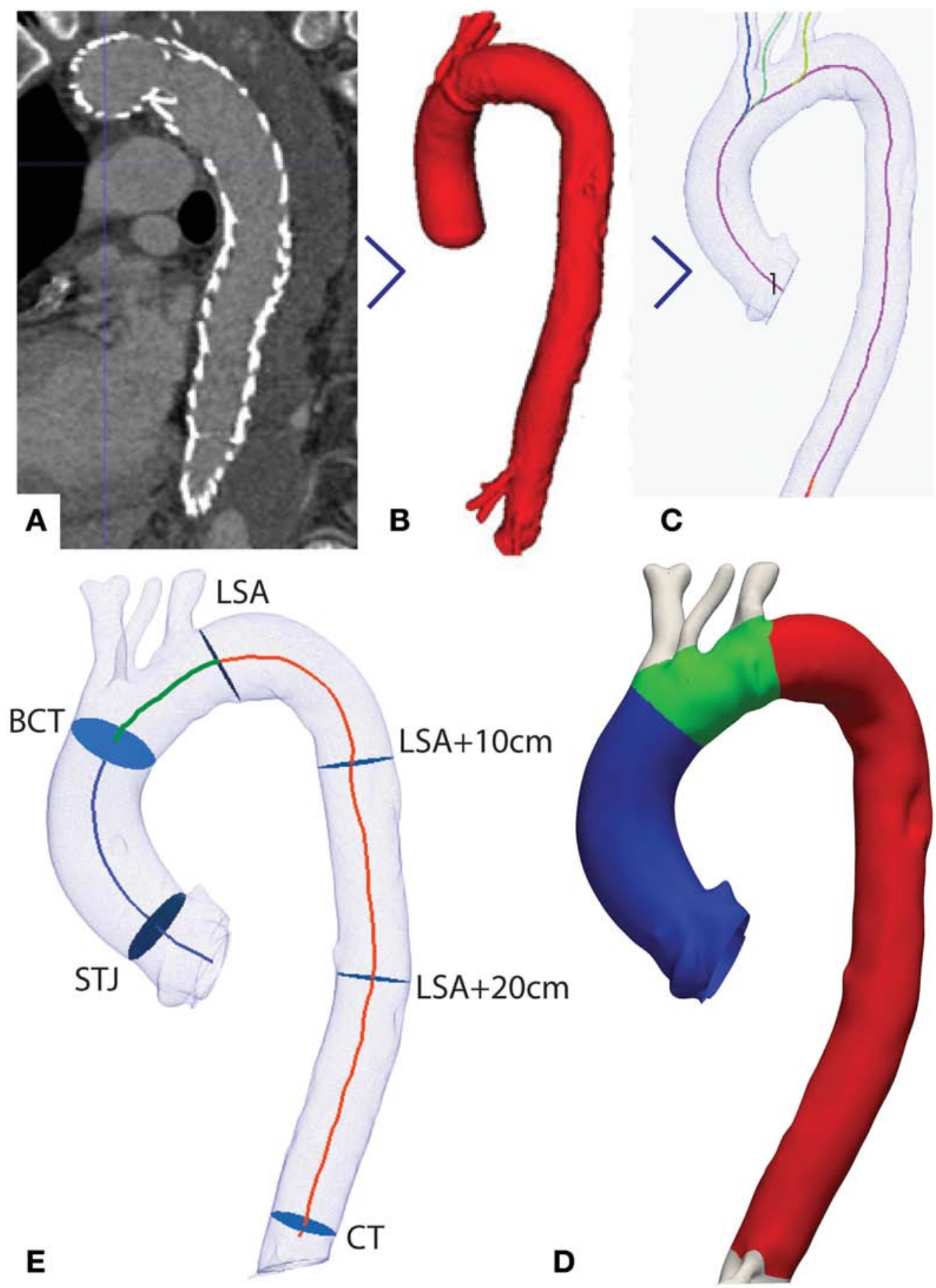

Figure 2. Workflow. Panel A. Cardiac-gated computed tomography angiography imaging provided dicom imaging sets during eight phases of the cardiac cycle. Panel B. The thoracic aorta was segmented at each phase, and three-dimensional reconstructed models were computed. Panel C. Center lumen lines were computed. Panels D and E. Longitudinal and circumferential segments were identified using side branch origins as anatomical landmarks, resulting into (Panel D) longitudinal segments (ascending aorta (purple), aortic arch (green), and descending aorta (red)) and (Panel E) circumferential levels (sinotubular junction (STJ), just before the origin of the brachiocephalic trunk (BCT), left subclavian artery (LSA), $10 \mathrm{~cm}(\mathrm{LSA}+10 \mathrm{~cm})$ and $20 \mathrm{~cm}$ (LSA $20 \mathrm{~cm})$ distal to the left subclavian artery, and celiac trunk (CT)). Lengths, areas, and diameters were then calculated by a custom-developed script [21]. 
org/10.12945/j.aorta.2017.16.042.sup.02). For enhanced vessel contrast, each patient received 90 $150 \mathrm{~mL}$ non-ionic contrast medium (lopromide, Schering, Berlin, Germany) followed by a $60-\mathrm{mL}$ saline chaser bolus.

2. Image segmentation: An imaging workstation was created to post-process the four-dimensional (4-D) image sets. Dedicated ITK-Snap software [20] was used to segment the thoracic aortic geometries of each phase both pre- and post-TEVAR. This software implements a 3-D active contour segmentation method, called snake evolution, which works on the closed surface of the aortic lumen. In addition, 3-D reconstruction of the different levels of interest was conducted for each phase (Figure 2B). The 3-D reconstructions for both TBAD patients before and after TEVAR are shown in Supplemental Figure 1 (see supplemental Figure 1 at http:// dx.doi.org/10.12945/j.aorta.2017.16.042.sup.01).

3. Quantification of dynamic aortic geometries: A customized script was developed combining methods implemented by Visualization Toolkit and Vascular Modeling Toolkit libraries [21]. This semi-automatic script extracts aortic geometries per segment/level of interest, starting with calculation of the vessel centerline of the true lumen. Subsequently, the spatial coordinates of the points of interest along the center lumen line are identified. The script then splits and computes the centerline at the origins of each side branch of interest (Figure 2C). Each center lumen line or segmentation slice was checked by an investigator and manually adjusted if needed.

4. Pulsatile longitudinal strain: Three segments of interest were identified consistent with the supra-aortic branch splitting: (1) the ascending aorta, starting at the sinotubular junction to the brachiocephalic trunk; (2) the aortic arch, extending from the brachiocephalic trunk to the LSA; and (3) the thoracic descending aorta, stretching from the LSA to the celiac trunk (Figure 2D). For each patient, pulsatile longitudinal strain was calculated as the difference between systolic and diastolic lengths divided by diastolic length.

5. Pulsatile circumferential strain: Aortic diameters and areas were measured at six different levels: the sinotubular junction, brachiocephalic trunk,
LSA, 10 and $20 \mathrm{~cm}$ distal to the LSA, and celiac trunk (Figure 2E). Diameters were measured in $360^{\circ}$ by calculating the distance between all points constituting the section of interest perpendicular to the center lumen line and then extracting the end-diastolic and end-systolic values, as previously described $[12,13]$. Aortic areas were measured by triangulating the particular section and summing the area of all obtained triangles. Pulsatile circumferential strain was computed as the difference between end-systolic and end-diastolic circumference divided by end-diastolic circumference.

\section{Intra- and Inter-Observer Variability}

Length and area measurements were acquired twice by the same investigator (C.T.) and blindly repeated by another investigator (F.N.) to allow for intra- and inter-observer variability.

\section{Statistical Analysis}

Data were analyzed with SPSS 22.0 (SPSS, Chicago, IL). Continuous data are presented as mean \pm standard deviation (SD) unless stated otherwise, and categorical data are given as counts (percentage). Intra- and inter-observer variability were calculated according to Bland and Altman. Data were considered normally distributed based on skewness and kurtosis Z-values between -1.96 and 1.96, a Shapiro-Wilk test $p>0.05$, and visualization of approximately normally distributed data with histograms. $p<0.05$ were considered statistically significant.

\section{Results}

Patient and procedural characteristics are shown in Table 1. The median interval from TEVAR to postoperative cardiac-gated CTA imaging was 0.4 months (range, 0.1-0.7).

\section{Aortic Length and Pulsatile Longitudinal Strain}

Aortic length and pulsatile longitudinal strain before and after TEVAR are shown in Table 2. In Patient 1 , the end-systolic length of the descending aorta decreased following TEVAR, while the ascending aorta 
Table 2. End-systolic aortic length and longitudinal strain for each patient.

\begin{tabular}{|c|c|c|c|c|c|}
\hline $\mathrm{Pt}$ & Location & Total Thoracic & Ascending & Arch & Descending \\
\hline & \multicolumn{5}{|c|}{ End-systolic length ( $\mathrm{mm})$} \\
\hline \multirow[t]{2}{*}{1} & Pre-TEVAR & $363.7 \pm 1.8$ & $80.7 \pm 1.6$ & $38.2 \pm 1.0$ & $246.3 \pm 1.5$ \\
\hline & Post-TEVAR & $361.1 \pm 2.2$ & $83.4 \pm 1.4$ & $42.6 \pm 1.8$ & $238.6 \pm 1.8$ \\
\hline \multirow[t]{2}{*}{2} & Pre-TEVAR & $391.2 \pm 3.1$ & $74.0 \pm 2.5$ & $42.3 \pm 1.4$ & $281.0 \pm 4.2$ \\
\hline & Post-TEVAR & $398.7 \pm 5.4$ & $80.6 \pm 3.8$ & $43.9 \pm 1.4$ & $275.1 \pm 1.8$ \\
\hline 3 & Control & $373.3 \pm 5.1$ & $73.0 \pm 3.5$ & $55.1 \pm 0.9$ & $246.3 \pm 1.4$ \\
\hline \multirow[t]{2}{*}{4} & Control & $336.3 \pm 2.3$ & $52.0 \pm 2.2$ & $50.1 \pm 0.8$ & $235.2 \pm 1.3$ \\
\hline & \multicolumn{5}{|c|}{ Longitudinal strain (\%) } \\
\hline \multirow[t]{2}{*}{1} & Pre-TEVAR & 1.4 & 5.0 & 8.6 & 1.5 \\
\hline & Post-TEVAR & 1.7 & 5.3 & 14.2 & 2.4 \\
\hline \multirow[t]{2}{*}{2} & Pre-TEVAR & 2.4 & 9.9 & 9.6 & 4.9 \\
\hline & Post-TEVAR & 4.4 & 16.8 & 9.5 & 1.7 \\
\hline 3 & Control & 4.5 & 14.0 & 3.9 & 1.7 \\
\hline 4 & Control & 2.1 & 12.9 & 4.2 & 1.7 \\
\hline
\end{tabular}

Values are shown as mean \pm SD, where appropriate. TEVAR $=$ thoracic endovascular aortic repair

and arch elongated. Patient 2 showed similar shortening of the descending aorta with elongation of the ascending aorta and arch after TEVAR.

Pulsatile longitudinal strain before and after TEVAR in the two acute TBAD patients are illustrated in Figure 3. Before TEVAR, pulsatile longitudinal strain throughout the thoracic aorta ranged from 1.5\% to $9.9 \%$ in the two TBAD patients and from $1.7 \%$ to $14.0 \%$ in the two control subjects, with mean pulsatile longitudinal strain of the total thoracic aorta of $1.9 \pm 0.5 \%$ and $3.3 \pm 1.2 \%$, respectively. In three of the four patients, pulsatile longitudinal strain was highest in the ascending aorta (9.9-14.0\%), decreasing downstream along the arch (3.9-9.6\%) and descending aorta (1.7-4.9\%).

After TEVAR, an increase of pulsatile longitudinal strain was observed proximal to the stent-graft in both TBAD patients $(65 \%$ increase in the arch of $\mathrm{Pa}$ tient 1 , and $70 \%$ increase in the ascending aorta of Patient 2). In the descending aorta, Patient 1, with a stent-graft length of $174 \mathrm{~mm}$, showed increased longitudinal strain (+60\%) following TEVAR. By contrast, Patient 2, with a longer stent-graft length of $235 \mathrm{~mm}$, showed decreased longitudinal strain (-65\%) in the descending aorta after TEVAR.
Aortic Diameter and Pulsatile Circumferential Strain

End-systolic diameter and circumferential strain are shown in Table 3. Overall, smaller diameters of the true lumen were observed in TBAD patients than in control subjects (from $10 \mathrm{~cm}$ distal to the LSA to the celiac trunk).

Figure 4 illustrates pulsatile circumferential strain before TEVAR, which ranged from $0.9 \%$ to $3.3 \%$ and was comparable among Patient 1 and the two control subjects. However, Patient 2, who presented with acute TBAD and patent false lumen, showed considerably higher circumferential strain at sections of the patent false lumen $(4.4 \%$ at $20 \mathrm{~cm}$ distal to the LSA and $6.2 \%$ at the celiac trunk).

After TEVAR, the true lumen expanded in sections adjacent to the stent-graft (brachiocephalic trunk and celiac trunk). Pulsatile circumferential strain also increased in sections adjacent to the stent-grafts, except for the distal adjacent section in Patient 2 (celiac trunk with patent false lumen), which decreased considerably (6.2\% before TEVAR vs. $2.5 \%$ after TEVAR). Diameter and area measurements within stented sections were deemed unreliable due to stent artifacts in the segmentations and were therefore excluded from this analysis. 


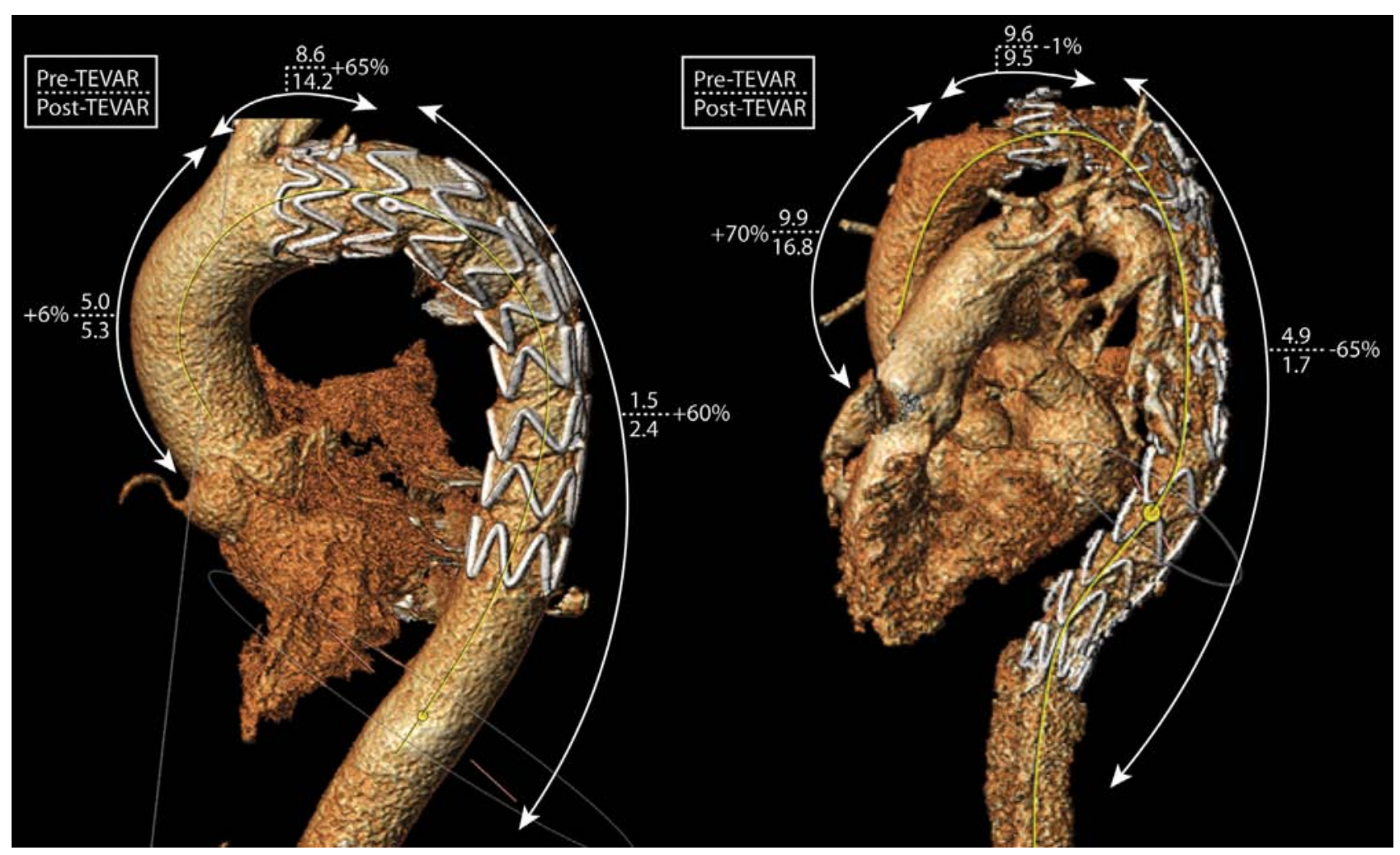

Figure 3. Pulsatile longitudinal strain before and after thoracic endovascular aortic repair thoracic endovascular aortic repair (TEVAR) in Patient 1 with acute Type B aortic dissection (TBAD) (left panel) and Patient 2 with ruptured acute TBAD and Marfan syndrome (right panel). Pre- and post-TEVAR strain values are shown as percentages. Relative strain changes were computed as the percentage increase or decrease of post-TEVAR strain relative to pre-TEVAR strain.

Table 3. End-systolic diameter and circumferential strain.

\begin{tabular}{|c|c|c|c|c|c|c|c|}
\hline & Location & STJ & BCT & LSA & $\mathrm{LSA}+10 \mathrm{~cm}$ & $\mathrm{LSA}+20 \mathrm{~cm}$ & CT \\
\hline & \multicolumn{7}{|c|}{ End-systolic diameter ( $\mathrm{mm})$} \\
\hline \multirow[t]{2}{*}{1} & Pre-TEVAR & $33.6 \pm 0.8$ & $36.3 \pm 0.4$ & $30.2 \pm 0.4$ & $23.7 \pm 0.3$ & $22.8 \pm 0.1$ & $24.2 \pm 0.3$ \\
\hline & Post-TEVAR & $33.2 \pm 0.7$ & $36.4 \pm 0.4$ & Stent & Stent & Stent & $27.2 \pm 0.6$ \\
\hline \multirow[t]{2}{*}{2} & Pre-TEVAR & $32.3 \pm 0.5$ & $31.7 \pm 0.4$ & $24.2 \pm 0.2$ & $20.5 \pm 0.5$ & $22.4 \pm 0.9$ & $19.8 \pm 1.3$ \\
\hline & Post-TEVAR & $32.9 \pm 0.8$ & $32.2 \pm 0.8$ & $24.9 \pm 0.6$ & Stent & Stent & $21.6 \pm 0.3$ \\
\hline 3 & Control & $34.3 \pm 1.4$ & $34.0 \pm 0.9$ & $31.4 \pm 1.1$ & $27.2 \pm 1.1$ & $26.5 \pm 1.1$ & $26.5 \pm 1.0$ \\
\hline 4 & Control & $34.2 \pm 0.7$ & $36.6 \pm 0.6$ & $28.2 \pm 0.5$ & $28.0 \pm 0.4$ & $27.4 \pm 0.2$ & $27.0 \pm 0.1$ \\
\hline & \multicolumn{7}{|c|}{ Circumferential strain (\%) } \\
\hline \multirow[t]{2}{*}{1} & Pre-TEVAR & 2.1 & 1.5 & 2.0 & 2.1 & 1.4 & 1.9 \\
\hline & Post-TEVAR & 2.2 & 1.6 & Stent & Stent & Stent & 3.2 \\
\hline \multirow[t]{2}{*}{2} & Pre-TEVAR & 2.4 & 1.5 & 1.9 & 2.8 & 4.4 & 6.2 \\
\hline & Post-TEVAR & 3.5 & 2.7 & 3.6 & Stent & Stent & 2.5 \\
\hline 3 & Control & 3.3 & 2.1 & 1.8 & 1.7 & 2.2 & 2.0 \\
\hline 4 & Control & 2.3 & 1.2 & 2.9 & 1.8 & 1.2 & 0.9 \\
\hline
\end{tabular}

Values are shown as mean $\pm \mathrm{SD}$, where appropriate. $\mathrm{BCT}=$ brachiocephalic trunk; $\mathrm{CT}=$ celiac trunk; $\mathrm{LSA}=$ left subclavian artery; $\mathrm{STJ}=$ sinotubular junction; TEVAR = thoracic endovascular aortic repair. 

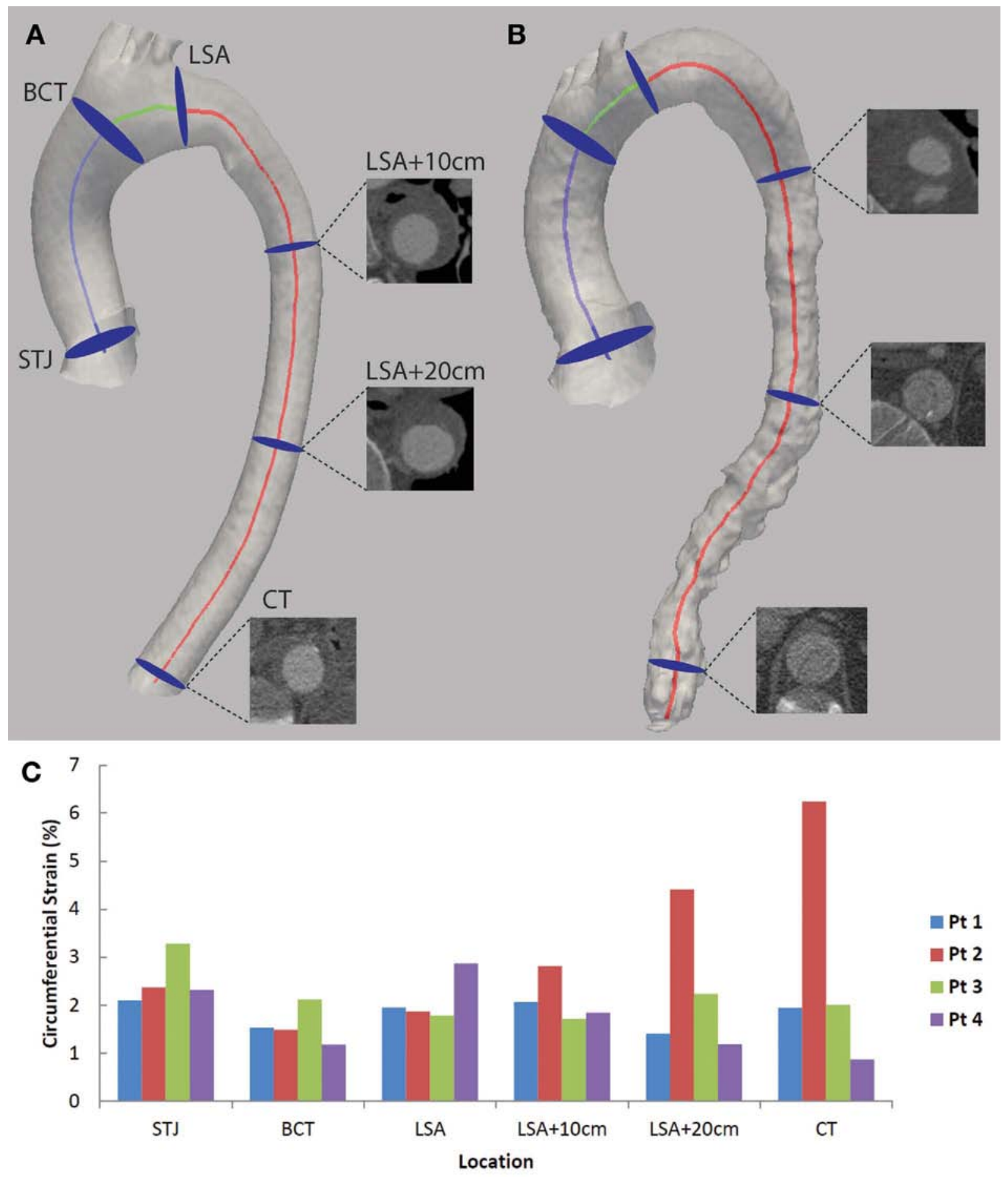

Figure 4. Panels $A$ and $B$. Pulsatile circumferential strain of (Panel A) Patient 1 with acute Type $B$ aortic dissection (TBAD) and thrombosed false lumen and (Panel B) Patient 2 with acute ruptured TBAD, Marfan syndrome, and patent false lumen at the LSA $20 \mathrm{~cm}$ and $C T$ levels. Panel $C$. Comparison of pulsatile circumferential strain among all patients. $B C T=$ brachiocephalic trunk; $C T=$ celiac trunk; $L S A=$ left subclavian artery; $L S A+10 \mathrm{~cm}=10 \mathrm{~cm}$ distal to the $L S A ; L S A+20 \mathrm{~cm}=20 \mathrm{~cm}$ distal to the $L S A ; S T J=$ sinotubular junction. 


\section{Intra- and Inter-Observer Variability}

For area changes, the intra-observer repeatability coefficient (RC) was $22.85 \mathrm{~mm}^{2}$. Mean differences between pre- and post-TEVAR area changes were smaller than the $R C s$, and linear regression analysis was non-significant $(p=0.31)$. Inter-observer repeatability showed an RC of $17.88 \mathrm{~mm}^{2}$, and mean differences between pre- and post-TEVAR area changes were smaller than the RCs. Linear regression analysis was non-significant $(p=0.46)$. These results indicate good intra- and inter-observer agreement.

For length changes, the RC for intra-observer repeatability was $2.78 \mathrm{~mm}$, and mean differences between pre- and post-TEVAR length changes were smaller than the RCs. Linear regression analysis was non-significant $(p=0.70)$. The $R C$ for interobserver repeatability was $2.84 \mathrm{~mm}$. Mean differences between pre- and post-TEVAR areas were smaller than the $\mathrm{RCs}$, and linear regression analysis was non-significant $(p=0.64)$. Again, these results indicate good intra- and inter-observer agreement.

\section{Discussion}

In both TBAD patients, TEVAR was followed by elevated pulsatile longitudinal strain proximal to the stent-graft. Moreover, TBAD patients showed lower pulsatile longitudinal strain of the total thoracic aorta before TEVAR compared with control subjects. Regarding pulsatile circumferential strain, Patient 1 , who presented with patent false lumen, showed higher strain of the true lumen when compared with Patient 2, who had a thrombosed false lumen. Pulsatile circumferential strain in control subjects ranged from $0.9 \%$ to $3.3 \%$ and was consistent along the thoracic aorta, which agrees with previous literature [22].

After TEVAR, we found increased pulsatile longitudinal wall strain proximal to the stent-grafts in both TBAD patients. Other authors suggest that increased pulsatile wall stress is associated with aortic wall fatigue, resulting in aneurysmal dilatation and increased risk of new entry tears at the proximal or distal end of the stent-graft or rupture [2-5]. Patients with fragile aortic walls, such as those with aortic dissection, are at particular risk for such sudden strain changes. In our study, Patient 2 developed aortic perforation due to a stent strut. In this patient, we observed a considerable increase in circumferential strain (1.9\% vs. 3.6\%) at the LSA after the initial TEVAR procedure. This elevated strain may have triggered the strut perforation. To better understand the pathophysiology of such complications, further studies are warranted to investigate pulsatile strain changes following TEVAR.

Pulsatile longitudinal strain was lower in TBAD patients than in older-aged control subjects $(1.4-1.7 \%$ vs. $2.1-4.5 \%)$, even though aging is associated with aortic stiffening $[8,22]$. This observation suggests that aortic stiffening is a risk factor for aortic dissection, although this remains to be confirmed by larger studies. Moreover, in Patient 2, we observed that stent-grafting of a longer segment was associated with a decrease in longitudinal strain, stiffening the descending aorta and potentially exerting adverse effects on cardiac function and geometry [10].

We noticed markedly high pulsatile circumferential strain at the level of patent false lumen in Patient 1. This was in contrast to the control subjects and Patient 2, who had a thrombosed false lumen. This observation emphasizes the potential role of variables such as dissection flap motion and thrombosis on prognosis. It also sheds light on differences between acute and chronic TBAD and underscores the importance of dynamic imaging to assess these risk factors and correctly size stent-grafts. Moreover, a substantial pulsatile circumferential strain may have implications for future device selection, as patients with more compliant aortas may benefit from more compliant stent-grafts to minimize mismatch.

Aortic stiffness is reportedly higher in Marfan patients [23]. We did not observe this in our study, most likely because our Marfan patient (Patient 2) presented with acute TBAD complicated by rupture. The movement of the dissection flap elevated the pulsatile circumferential strain before TEVAR. In addition, this patient was younger than the two control subjects, and younger age is associated with higher strain [22].

To continuously improve treatment outcomes of TBAD, it is important to obtain data on the biomechanical behavior of dissected aortas. In particular, promising advancements in computational fluid dynamics offer unique insights into complex vascular pathologies [8]. However, such computational techniques depend on clinically measured data to serve 
as boundary conditions, which are only sparsely available for TBAD. Thus, this study provides preliminary data on dissection flap strain.

The main limitation of this study is the small cohort, as we were only able to include two TBAD patients and two control subjects. Therefore, our results cannot be generalized and should be considered descriptive. Moreover, we were unable to report circumferential strain within stented segments due to artifacts. More systematic studies are needed to elucidate the effects of aortic stent-grafts on the physiologic function of the aorta. Finally, with advancements in medical imaging, pixel spacing and the number of equidistant time steps during the cardiac cycle could be improved and should be further investigated.

In conclusion, this study represents an initial investigation on pulsatile aortic strain in patients with acute TBAD before and after TEVAR. We observed considerably higher pulsatile circumferential strain in a case of patent false lumen compared with thrombosed false lumen. After TEVAR, both TBAD patients showed increased pulsatile longitudinal strain proximal to the stent-graft, which is known to be associated with an increased risk of aortic dissection. These observations may motivate future research to assess the biomechanical impact of TEVAR in order to continuously improve treatment outcomes for patients with TBAD.

\section{Acknowledgments}

Prof. F. Auricchio and Dr. M. Conti acknowledge the support of Ministero dell'Istruzione, dell'Università e della Ricerca (No. 2010BFXRHS), ERC Starting Grant through the Project ISOBIO: Isogeometric Methods for Biomechanics (No. 259229), and the iCardiocloud project by Cariplo Foundation (No. 2013-1779) and Lumbardy Region (No. 42938382; No. 46554874).

\section{Conflict of Interest}

The authors have no conflicts of interest relevant to this publication.

\section{Comment on this Article or Ask a Question}

\section{References}

1. Svensson LG, Kouchoukos NT, Miller DC, Bavaria JE, Coselli JS, Curi MA, et al. Expert consensus document on the treatment of descending thoracic aortic disease using endovascular stent-grafts. Ann Thorac Surg. 2008;85:S1-41. DOI: 10.1016/j.athoracsur. 2007.10.099

2. Dong ZH, Fu WG, Wang YQ, Guo DQ, Xu X, Ji $Y$, et al. Retrograde type $A$ aortic dissection after endovascular stent graft placement for treatment of type B dissection. Circulation. 2009;119:735-741. DOI: 10.1161/ CIRCULATIONAHA.107.759076

3. Dong Z, Fu W, Wang Y, Wang C, Yan Z, Guo $D$, et al. Stent graft-induced new entry after endovascular repair for Stanford type B aortic dissection. J Vasc Surg. 2010;52:14501457. DOI: 10.1016/j.jvs.2010.05.121

4. Huang W, Luo S, Luo J, Liu Y, Fan R, Xue L, et al. Perioperative aortic dissection rupture after endovascular stent graft placement for treatment of type B dissection. Chin Med J (Engl). 2013;126:1636-1641. PMID: 23652043

5. Raaz U, Zöllner AM, Schellinger IN, Toh R, Nakagami F, Brandt $M$, et al. Segmental aortic stiffening contributes to experimental abdominal aortic aneurysm development.
Circulation. 2015;131:1783-1795. DOI: 10.1161/CIRCULATIONAHA.114.012377

6. Brekken R, DahlT, Hernes TAN, Myhre HO. Reduced strain in abdominal aortic aneurysms after endovascular repair. J Endovasc Ther. 2008;15:453-461. DOI: 10.1583/07-2349.1

7. Kleinstreuer C, Li Z, Basciano CA, Seelecke $S$, Farber MA. Computational mechanics of Nitinol stent grafts. J Biomech. 2008;41:23702378. DOI: 10.1016/j.jbiomech.2008.05.032

8. Roccabianca $S$, Figueroa $C A$, Tellides G, Humphrey JD. Quantification of regional differences in aortic stiffness in the aging human. J Mech Behav Biomed Mater. 2014;29:618-634. DOI: 10.1016/j. jmbbm.2013.01.026

9. Tzilalis VD, Kamvysis $D$, Panagou $P$, Kaskarelis I, Lazarides MK, Perdikides T, et al. Increased pulse wave velocity and arterial hypertension in young patients with thoracic aortic endografts. Ann Vasc Surg. 2012;26:462-467. DOI: 10.1016/j. avsg.2011.06.021

10. Takeda $Y$, Sakata $Y$, Ohtani T, Tamaki S, Omori Y, Tsukamoto Y, et al. Endovascular aortic repair increases vascular stiffness and alters cardiac structure and function. Circ J. 2014;78:322-328. DOI: 10.1253/circj.

\section{CJ-13-0877}

11. van Prehn J, Vincken KL, Sprinkhuizen SM, Viergever MA, van Keulen JW, van Herwaarden JA, et al. Aortic pulsatile distention in young healthy volunteers is asymmetric: analysis with ECG-gated MRI. Eur J Vasc Endovasc Surg. 2009;37:168-174. DOI: 10.1016/j.ejvs.2008.11.007

12. van Prehn J, Vincken $\mathrm{KL}$, Muhs BE, Barwegen GKW, Bartels LW, Prokop M, et al. Toward endografting of the ascending aorta: insight into dynamics using dynamic cine-CTA. J Endovasc Ther. 2007;14:551560. DOI: $10.1177 / 152660280701400418$

13. Muhs BE, Vincken KL, van Prehn J, Stone MKC, Bartels LW, Prokop M, et al. Dynamic cine-CT angiography for the evaluation of the thoracic aorta; insight in dynamic changes with implications for thoracic endograft treatment. Eur JVasc Endovasc Surg. 2006;32:532536. DOI: 10.1016/j.ejvs.2006.05.009

14. Khanafer K, Duprey A, Zainal M, Schlicht $M$, Williams $D$, Berguer R. Determination of the elastic modulus of ascending thoracic aortic aneurysm at different ranges of pressure using uniaxial tensile testing. J Thorac Cardiovasc Surg. 2011;142:682-686. DOI:10.1016/j.jtcvs.2010.09.068 
15. Beller CJ, Labrosse MR, Thubrikar MJ, Robicsek F. Role of aortic root motion in the pathogenesis of aortic dissection. Circulation. 2004;109:763-769. DOI: 10.1161/01. CIR.0000112569.27151.F7

16. Robicsek F, Thubrikar MJ. Hemodynamic considerations regarding the mechanism and prevention of aortic dissection. Ann Thorac Surg. 1994;58:1247-1253. PMID: 7944800

17. Bell V, MitchellWA, Sigurðsson S, Westenberg JJM, Gotal JD, Torjesen AA, et al. Longitudinal and circumferential strain of the proximal aorta. J Am Heart Assoc. 2014;3:e001536. DOI: 10.1161/JAHA.114.001536

18. Jonker FHW, van Keulen JW, Schlosser FJV, Indes JE, Moll FL, Verhagen HJM, et al. Thoracic aortic pulsatility decreases during hypovolemic shock: implications for stentgraft sizing. J Endovasc Ther. 2011;18:491496. DOI: 10.1583/10-3374.1
19. van Keulen JW, Moll FL, Jahrome AK, van Herwaarden JA. Proximal aortic perforation after endovascular repair of a type B dissection in a patient with Marfan syndrome. J Vasc Surg 2009;50:190-192. DOI: 10.1016/j.jvs.2009.01.045

20. Yushkevich PA, Piven J, Hazlett HC, Smith RG, Ho S, Gee JC, et al. User-guided 3D active contour segmentation of anatomical structures: significantly improved efficiency and reliability. Neuroimage. 2006;31:1116-1128. DOI: 10.1016/j.neuroimage.2006.01.015

21. Trentin C, Faggiano $E$, Conti M, Auricchio F. An automatic tool for thoracic aorta segmentation and 3D geometric analysis. 2015 9th International Symposium on Image and Signal Processing and Analysis. Accessed on September 9, 2015 DOI: 10.1109/ISPA.2015.7306033

22. Morrison TM, Choi G, Zarins CK, Taylor
CA. Circumferential and longitudinal cyclic strain of the human thoracic aorta: age-related changes. J Vasc Surg. 2009;49:1029-1036. DOI: 10.1016/j. jvs.2008.11.056

23. Grillo A, Pini A, Marelli S, Gan L, Giuliano $A$, Trifirò $G$, et al. 5B.05: Marfan syndrome: assessment of aortic dissection risk by analysis of aortic viscoelastic properties. J Hypertens. 2015;33:e67. DOI: 10.1097/01. hjh.0000467528.12746.36

Cite this article as: Nauta $\mathrm{FJH}$, Bogerijen GHW, Conti M, Trentin C, Moll FL, Herwaarden JA, Auricchio F, Trimarchi S. Impact of Thoracic Endovascular Repair on Pulsatile Aortic Strain in Acute Type B Aortic Dissection. AORTA (Stamford). 2017;5(2):42-52. DOI: https://doi. org/10.12945/j.aorta.2017.16.042

\section{EDITOR'S COMMENT}

The provocative preliminary observations by Foeke and colleagues should stimulate our attention. We tend to focus on the stented zone after TEVAR, with perhaps less than optimal consideration for the non-stented zone. These investigators, in a very preliminary study, show that having a descending stent graft substantially increases the stretch of the ascending or arch zones with each heartbeat. They speculate, plausibly, that this increased stretch may be harmful and may contribute to early and late complications in the aorta out- side the stent zone. This may be especially pertinent vis-a-vis the troublesome, and often lethal, occurrence of retrograde ascending aortic dissection following descending aortic stent grafting. While the number of patients studied is very small, the authors describe the technical methods of analysis clearly; hopefully, their posing these important questions and laying the requisite computational methods will stimulate more extensive studies of this important topic. 